\title{
Prática Colaborativa de Ensino Híbrido: uso de smartphone no Design de aula de inglês
}

\author{
Willian Rufato da Silva* \\ Marilene Santana dos Santos Garcia**
}

\section{Resumo}

O objetivo deste estudo é aprofundar o conhecimento de como dispositivos móveis transformam-se em plataformas para ancorar atividades educacionais híbridas, ocupando espaços mediados por tecnologias no espaço de sala de aula. Na prática, trata-se do uso de smartphones, celulares e tablets para envolver alunos e professores em atividades que qualifiquem esses contextos de aprendizagem. Esse estudo apresenta orientações sobre algumas práticas pedagógicas colaborativas aplicadas com dispositivos móveis e avalia de que forma os professores se sentem com tais aplicações. A pesquisa é de natureza descritiva, com abordagem qualitativa, em que cinco professores foram convidados a participar de atividades pedagógicas híbridas, com uso de celulares móveis e do aplicativo Duolingo, trabalhado na disciplina de língua inglesa em uma escola pública. Como resultados constatou-se que práticas com Mobile Learning abrem opor-

* Mestre em Educação e Novas Tecnologias (2020) e Especialista em Educação Especial e Atendimento inclusivo (2015) pelo Centro Universitário Internacional (Uninter), Graduado em Letras Português - Inglês pela Universidade Tuiuti do Paraná (2011). Assessor Pedagógico, escritor de Língua Inglesa na Positivo Soluções Didáticas e Especialista em Sala de Recursos Multifuncional pela rede estadual do Paraná.

** É mestre em linguística pela Universidade Estadual de Campinas (Unicamp) e doutora em letras pela USP, com bolsa de pesquisa nas Universidades de Oldenburg e de Freiburg (Alemanha). Graduada em Letras (alemão, português e linguística) pela Universidade de São Paulo (USP) e Pedagogia pelo Centro Universitário Internacional Uninter). Concluiu pós doutorado pela Pontifícia Universidade Católica de São Paulo (PUC-SPTIDD) em tecnologias da inteligência e design digital. Atualmente é professora na PUC-SP. 
tunidades para o uso de recursos tecnológicos digitais, como objetos de aprendizagem, aplicativos, músicas, micro-conteúdos, sites entre outros, com apoio de metodologias ativas e quando bem contextualizadas, melhoram competências socioemocionais e culturais e trazem mais motivação à aprendizagem. Paa tanto, foram definidos dez (10) indicadores para realização dessas atividades híbridas. Foram tomados os seguintes autores para apoios teóricos basais: Starker \& Horn (2015), Filatro (2018), Garcia (2016), Moran (2018), Bacich (2018), Ladd (2009), Campbell (2012), Cechinel (2017).

Palavras-chave: Mobile Leaning. Educação. Metodologias Ativas. Ensino Híbrido

\section{Collaborative practice of blended learning: use of smartphone in the design of English as foreign language classes}

\section{Abstract}

The aim of this study is to deepen the knowledge of how mobile devices become platforms to anchor hybrid educational activities, occupying spaces mediated by technologies in the classroom space. In practice, it is about the use of devices such as smartphones, mobile phones and tablets to involve students and teachers in activities that qualify these learning contexts. This study presents guidance on some collaborative pedagogical practices applied with mobile devices and assesses how teachers feel about such applications. The research is descriptive in nature, with a qualitative approach, in which five teachers were invited to participate in hybrid pedagogical activities, using mobile phones and the Duolingo application, worked in the English language discipline in a public school. As results, it was found that practices with Mobile Learning open opportunities for the use of digital technological resources, such as learning objects, applications, music, micro-content, websites, among others, with the support of active methodologies and when well contextualized, improve socio-emotional and cultural skills and bring more motivation to learning. However, ten (10) indicators were defined to perform these hybrid activities. The following authors were taken for baseline theoretical support: Starker \& Horn (2015), Filatro (2018), Garcia (2016), Moran (2018), Bacich (2018), Ladd (2009), Campbell (2012), Cechinel (2017).

Keywords: Mobile learning. Education. Active Methodologies. Blended Learning 


\section{Práctica colaborativa de blended learning: uso de smartphone en el diseño de clases de inglés como lengua extranjera}

\section{Resumen}

El objetivo de este estudio es profundizar en el conocimiento de cómo los dispositivos móviles se convierten en plataformas para anclar actividades educativas híbridas, ocupando espacios mediados por tecnologías en el espacio del aula. En la práctica, se trata del uso de dispositivos como teléfonos inteligentes, teléfonos móviles y tabletas para involucrar a los estudiantes y profesores en actividades que califican estos contextos de aprendizaje. Este estudio presenta orientación sobre algunas prácticas pedagógicas colaborativas aplicadas con dispositivos móviles y evalúa cómo se sienten los profesores acerca de este tipo de aplicaciones. La investigación es de carácter descriptivo, con un enfoque cualitativo, en el que cinco profesores fueron invitados a participar en actividades pedagógicas híbridas, utilizando teléfonos móviles y la aplicación Duolingo, trabajó en la disciplina del idioma inglés en una escuela pública. Como resultado, se encontró que las prácticas con Mobile Learning abren oportunidades para el uso de recursos tecnológicos digitales, como el aprendizaje de objetos, aplicaciones, música, microcontenidos, sitios web, entre otros, con el apoyo de metodologías activas y cuando están bien contextualizados, mejoran las habilidades socioemocionales y culturales y aportan más motivación al aprendizaje. Sin embargo, se definieron diez (10) indicadores para realizar estas actividades híbridas. Los siguientes autores fueron tomados para el soporte teórico de referencia: Starker \& Horn (2015), Filatro (2018), Garcia (2016), Moran (2018), Bacich (2018), Ladd (2009), Campbell (2012), Cechinel (2017).

Palabras clave: Mobile Learning. Educación. Metodologías activas. Prácticas híbridas

\section{Introdução}

A tecnologia móvel provoca impactos importantes na maneira como a sociedade trabalha, aprende e se diverte, de forma que se tornou parte integrante da vida moderna em todo o mundo. Os celulares inteligentes são dispositivos de comunicação e informação, com rápido crescimento e expansão no seu uso e aplicações (CAMPBELL, 2006). Atualmente, dependendo de sua configura- 
ção, a maioria dos celulares têm funcionalidades como as de um computador (FILATRO, 2017).

O conceito de mobile learning não se restringir apenas aos aplicativos para celular para alguma finalidade de ensino e aprendizagem. Por meio dessa modalidade abrem-se novas oportunidades pedagógicas com relação à comunicação entre professores e estudantes, permitindo, sobretudo, levar ações educacionais a locais de difícil acesso, onde não existem escolas ou professores, ou onde ocorram limitações no uso de objetos de aprendizagem on-line, recursos e materiais didáticos, podendo-se aplicar as suas funcionalidades digitais visando a tais finalidades (WALKER 2007, FILATRO 2017, GARCIA 2017, MATTAR 2018).

É importante destacar que a aprendizagem móvel relaciona-se às metodologias ativas, principalmente em função da forma como são exploradas em ambientes educacionais.

Os princípios das metodologias ativas visam a abrir espaço para o desempenho protagonista do estudante, na medida em que este é orientado a desenvolver atividades de modo participativo, reflexivo, participando de uma experiência ou de um projeto, de forma a poder desenhar, co-criar, usar sua criatividade, com acompanhamento do professor, cujas ações são, preferencialmente, negociadas, dinamizadas, contextualizadas e reconfiguradas socialmente, em todas as etapas que envolvem essa modalidade de ensino/ aprendizagem ( MORAN 2018; BACICH, 2018; GARCIA, 2018),

Assim, este artigo busca apontar algumas práticas educacionais, em que se conseguiu aplicar e testar atividades de ensino híbrido com uso de celulares inteligentes em sala de aula para o ensino do inglês em uma escola pública situada em Curitiba-Paraná. O objetivo dessa orientação prática tem a ver com a necessidade de se planejar de forma criativa, coerente e otimizada as atividades propostasm em ambiente presencial com celulares inteligentes. 


\section{Internet e mobile-learning como plataformas educacionais}

A internet traz em seus espaços virtuais a possibilidade de interação entre os indivíduos aprendizes e seus pares. A integração das tecnologias digitais na educação precisa ser feita de modo criativo e crítico, buscando desenvolver a autonomia e a reflexão dos seus envolvidos, para que eles não sejam apenas receptores de informações (BACICH, TANZI NETO, TREVISANI, 2015).

Santaella (2015) destaca que são notáveis os avanços trazidos para a educação, em todas as suas formas, pelo desenvolvimento da tecnologia computacional. Com seus dispositivos móveis modifica-se a relação entre os atores principais do processo de ensino aprendizagem, professores e estudantes, e a interação com o conhecimento.

Assim, telefones celulares, laptops e smartphones, por exemplo, tornam-se importantes dispositivos mediadores do processo de ensino e aprendizagem, abrindo possibilidades para propostas metodológicas que venham a engajar os indivíduos, com foco em atividades mais participativas, cujo estado de mobilidade intensa também leve a afetar tais relações de aprendizagem.

Segundo Fredricks (2004), o engajamento escolar é uma construção multidimensional, constituída de componentes comportamentais, emocionais e cognitivos. Para Santos (2013), “engajamento escolar é um conceito que se refere à relação que o estudante estabelece com as atividades escolares que lhes são propostas" (p. 31). No entanto, para Ladd e Dinella (2009), essa relação, não deve se restringir à presença física na escola ou mesmo na sala de aula, mas visa a envolver o engajamento comportamental, emocional e cognitivo como determinantes potenciais para a aprendizagem e o sucesso escolar.

O ensino híbrido é uma das decorrências da aplicação do mobile learning em sala de aula. Para Bacich (2015), no ensino híbrido não existe uma única forma de aprender e a aprendizagem é um processo contínuo, que ocorre de diferentes formas e em diferentes espaços. 
Para Filatro (2017) podemos salientar que o protagonismo na aprendizagem via mobile learning ainda é um processo em desenvolvimento e que, cada vez mais, tende a extrair dos objetos digitais e móveis de aprendizagem, na forma de aplicativos, possibilidades mais responsivas para quem aprende tanto em ambientes combinados, os chamados híbridos ou combinados com mediações presenciais e digitais, como em ambientes da autoaprendizagem e informalidade, em que se acessam objetos de aprendizagem diversificados, procurando alcançar, da mesma maneira, diferentes tipos de conhecimento.

Nesse contexto, a aprendizagem com Mobile Learning torna possível o acesso, em tempo real, pelos chamados espaços virtuais, ao conhecimento sistematizado, ampliando-se para uma pedagogia para além das salas de aula, tornando o processo de ensino aprendizagem algo mais interativo, de caráter colaborativo.

De acordo com Garcia (2018):

À medida que os dispositivos de comunicação foram tornando-se cada vez mais portáteis e, ao mesmo tempo, receberam soluções de tecnologia smart (momento em que se aliaram à conectividade), iniciou-se a chamada era dos mobiles, estendendo-se a conceitos como sociedade mobile e mesmo mobile hominis (homem mobile). Dessa maneira, tais dispositivos não só passaram a oferecer novas oportunidades para se comunicar, interagir, produzir, compartilhar, colaborar e acessar conteúdos a qualquer hora e a partir de qualquer lugar, mas também tornaram seus usuários cidadãos on-line, visíveis, rastreados, ligados e acessíveis pelos inúmeros canais comunicativos e pelos aplicativos disponíveis. (GARCIA, 2018, p. 9)

Sendo assim, o mobile learning deve ser visto como uma possibilidade de promover diálogos para a aprendizagem que se beneficiam da mobilidade em espaços e tempos, bem como do próprio perfil já alcançado dos estudantes em termos de habilidades e competências, pois, quando os estudantes chegam à escola, já estão envoltos em inúmeras informações, dominam letramentos digitais, ou seja, sabem interagir com diversos recursos tecnológicos, devido à facilidade de acesso e às experiências contínuas nesse âmbito além dos muros da escola. 


\section{Recursos para práticas educacionais com dispositivos móveis}

Nesse sentido, é relevante mencionar alguns recursos desse mundo digital e mobile para os processos de ensino e aprendizagem. Destacamos algumas delas:

- Vídeo-aulas: gravadas em estúdio, geralmente de curta duração, especialmente para consumo em telas menores e em pequenos intervalos de tempo. $\mathrm{O}$ fornecedor pode disponibilizá-las apenas on-line, o que limita o acesso, ou em modo off-line.

- Aplicativos: desenvolvidos sob medida para dispositivos móveis, visam normalmente a alguma forma de serviço ou de aprendizagem. Eles podem combinar questionários interativos, conteúdo multimídia e mecanismos de interação entre os usuários.

- Gamificação: atividades que aproveitam mecanismos diversos, como personagens, pontuação, prêmios e níveis de dificuldade, a fim de dinamizar o processo de aprendizagem.

- Livros digitais: podem ser simples arquivos estáticos em PDF ou podem explorar ao máximo os recursos multimídia disponíveis em dispositivos móveis, incluindo a leitura vídeos, áudios, etc.

- Cursos online: Os cursos on-line orientam para o conceito de e-learning, evidenciam-se como uma modalidade de ensino a distância, utilizada na orientação de aprendizagens por meio de mídia eletrônica e digital.

- Redes Sociais acadêmicas: formas de divulgação científica, contando inclusive com a adesão de pesquisadores e docentes, diretores e organizadores de repositórios institucionais. Essas redes sociais acadêmicas servem também para promover a divulgação de resultados de pesquisas, a comunicação e colaboração entre pesquisadores afins, também servem como bases de dados 
para recuperar artigos, pois uma característica comum das redes sociais acadêmicas é a possibilidade de depositar e compartilhar os artigos de sua autoria.

A utilização do Mobile Learning no ambiente profissional e no acadêmico, como citado nos items acima, demonstra a forte relevância deste potencial para melhorar a performance dos estudantes (GARCIA 2018, MORAN 2018, BACICH 2018, FILATRO 2018, MAT'TAR 2018).

Contudo, tornam-se necessárias propostas de atividades que não só aproveitem tais acessos e essas habilidades digitais, mas que também possam propiciar sua integração e expansão (FILATRO, 2017, MATAR (2018) PONS (2019), GARCIA (2019). Assim, ainda é um processo inovador que busca a melhoria da qualidade da aprendizagem.

\section{Metodologia}

A presente investigação parte de uma proposta metodológica identificada como observação-participante, uma vez que o pesquisador atuou também como professor titular da disciplina de Língua Inglesa na instituição educacional pública, para a qual foram ofertadas as práticas híbridas, com o apoio de duas especialistas de língua inglesa e duas especialistas em tecnologias educacionais.

Conforme Gil (1999, p. 110), a observação é um “[...] elemento fundamental para a pesquisa [...] chega a ser mesmo considerada como método de investigação.”, devido aos graus de exigência e que requer estar precisamente definida em termos de procedimento. Mas, vista como técnica, esta implica em:

[...] coleta de dados para conseguir informações e utiliza os sentidos na atenção de determinados aspectos da realidade. Não consiste apenas em ver e ouvir, mas também em examinar fatos e ou fenômenos que se deseja estudar (MARCONI; LAKATOS, 1999, p. 90).

Da mesma forma, a presente pesquisa enquadra-se em uma abordagem descritiva e qualitativa. 


\section{Procedimentos metodológicos e grupo estudado}

Participaram da pesquisa 100 (cem) alunos oriundos de um dos maiores colégios públicos da cidade de Curitiba - PR, que tiveram a disciplina de Língua Inglesa ofertada no contexto híbrido estruturado no segundo semestre de 2019, baseando-se no modelo de rotação por estações, a partir da fundamentação prático- teórica de Starker \& Horn (2015). As atividades foram realizadas num período de quarenta dias, em duas aulas de uma hora por semana.

O modelo de Rotação por Estações é uma forma de organização de espaços em sala de aula para que os estudantes possam realizar diferentes atividades. Tais espaços são denominados estações. A rotação ocorre por conta da realização de diferentes atividades em cada uma dessas estações dentro de um determinado período de tempo e com o uso determinados materiais didáticos e acesso digital.

Cada estação teve um objetivo de aprendizagem específico, estando relacionados à finalidade da aula. O tempo de realização das atividades em cada uma das estações é pré-estabelecido no planejamento, cumprindo finalidades pedagógicas e usando diferentes recursos, de modo que todos os alunos consigam passar por todas as estações pedagógicas. Para a participação em cada estação, são apresentadas claramente as instruções para realizar a atividade. As estações são independentes umas das outras. Contudo, promovem a conclusão de objetivos separados, para que no final possam se completar.

Para a referida pesquisa, o espaço de sala de aula era previamente preparado para cada dia de aula numa sala interativa, equipada com lousa digital, notebooks, mesas redondas, cadeiras, materiais lúdicos e pedagógicos como: livros didáticos, dicionários; cartolina, papel sulfite para as atividades impressas, conexão com internet banda larga, smartphone dos próprios alunos com o aplicativo Duolingo baixado.

\section{Descritivo da aplicação híbrida com mobile na rotação por estações}

Os elementos principais contemplados na rotação por estações foram: ler, escrever, falar e ouvir tanto de forma analógica como 
também digital, fazendo o uso do próprio smartphone de cada aluno em sala de aula; por ser um modelo de ensino híbrido, algumas das estações foram feitas de forma online para que os estudantes pudessem ter mais autonomia, sendo que nesta pesquisa a principal estação online era a que utilizava o aplicativo educacional Duolingo.

As estações possuíam a seguinte funções pautadas em suas atividades e disponibilização de recursos:

Estação 1 - Watch and Listening Area: Nesta estação os alunos assistiam a vídeos e escutavam músicas no YouTube em consonância com o conteúdo da aula.

Estação 02 - Writen Area: Neste espaço da mesma sala os alunos realizavam atividades escritas feitas em computadores ou até mesmo na versão impressa.

Estação 03: Autonomy Area: Nesta estação os alunos praticavam o inglês fazendo uso dos seus próprios smartphones utilizando o aplicativo Duolingo sem auxílio dos colegas.

Estação 04: Conversation and feedback: Nesta área os alunos conversam com o professor sobre o tema da aula e tiravam suas dúvidas referentes ao tema central da aula.

\section{Resultados}

\section{Relato de experiência dos professores no processo de observação}

Na observação-participante realizada, uma das professoras do $8^{\circ}$ ano relatou que o uso de metodologias ativas com o aplicativo Duolingo na prática pedagógica foi benéfico com os alunos em sala de aula por meio do uso de recursos tecnológicos digitais e do trabalho colaborativo proposto nas esstações e fora dela. No relato da professora fica evidente a sua percepção acerca da mediação docente:

Diante de uma responsabilidade imensa [...] e de uma diversidade cultural que cada aluno carrega em seu núcleo familiar, já comecei a observar que antes de ensinar a língua inglesa é necessário aprender a conviver com o idioma, a respeitar e ter empatia pelo outro e entendi que eu como profes- 
sora, nesse turbilhão das mídias digitais, teria que mediar essa aprendizagem e que seria um processo continuo e muita colaboração (professora 1).

Em relação ao trabalho em equipes, ela descreve as dificuldades iniciais de socialização dos alunos:

Realmente essa relação em grupos, bem como a disposição circular das estações de trabalho propostas na rotação por estações, não é simples de executar mas reflete os novos valores de uma sociedade, desenvolvendo a construção de um cidadão mais conectado e reflexivo com o próximo (professora 1).

O relato da professora evidencia suas dificuldades na construção colaborativa e dialógica com o aluno. Esta evidencia a necessidade de usar criatividade ao organizar a rotação por estação e que 'construiu junto com os alunos' algumas regras para possibilitar o desenvolvimento do trabalho pedagógico em grupo, de forma a solucionar os conflitos. Essa mediação possibilitou que o objetivo do trabalho em equipe, que é a aprendizagem colaborativa, fosse atingido, conforme destacou:

Essa socialização entre grupos com o Duolingo, ao longo das aulas transcorrendo, trouxe fortalecimento nas relações entre nós todos. Fez com que os adolescentes com mais dificuldades linguísticas pudessem estreitar mais laços de amizades e também mostrar o quão rico é a troca ao colaborar para um único objetivo. (professora 2)

A outra professora especialista participante também relatou que a tecnologia digital não era usada em suas aulas para realização de pesquisas, desmistificando para própria profissional, por meio da aplicação do projeto de mestrado, que o uso dos dispositivos móveis podem, de fato, contribuir para suas práticas de sala de aula, pois quando o professor interage com os recursos tecnológicos, os alunos também aprendem a compreender a relevância da seleção adequada de conteúdos mais qualitativos ao aprender o inglês e 
com efeito pedagógicos, como nas atividades que foram propostas que inclui todos com ou mais habilidades comunicacionais (ler, escrever, falar e ouvir) na língua inglesa durante o projeto.

\section{Indicadores}

Por meio das observações-participativas e registros realizados pelos professores durante as aulas e de questionários prévios e posteriores aplicados com as turmas dos estudantes desta investigação sobre as práticas híbridas ofertadas, foi possível identificar os seguintes indicadores:

a) Concentração: A concentração dos alunos dentro da sala de aula foi satisfatória para os professores. Foi observado que os alunos têm acesso as mídias sociais e querem continuar a utilizá-las mesmo durante as aulas, o que dificultou algumas vezes o trabalho dos educadores que ainda não as utilizavam.

\section{b) Mobilidade:}

Nas práticas desta pesquisa foi adotada a modalidade de m-learning pelo uso do aplicativo Duolingo. Considerando-se o lado social e institucional do colégio pesquisado, inserir esta metodologia em suas estratégias de aprendizagem pode representar uma diminuição significativa de custos, pois as atividades são feitas remotamente, sem precisar ocupar com espaço físico, utilizar o tempo dos alunos/colaboradores para participar da dinâmica, nem com deslocamento para outros lugares e compra de materiais. Além disso, todos os alunos possuíam um smartphone e em condições para participar dessas atividades.

\section{c) Blended Learning (ensino híbrido):}

Ao acrescentar tecnologia nas aulas presenciais, foi necessário repensar todo o planejamento e ter em mente que professor e alunos assumissem novos papéis no processo de ensino-aprendizagem. Foram considerados a infraestrutura necessária, a capacitação dos professores, os métodos avaliativos, dentre outras características. Houve o cuidado de definir o tipo de tecnologia usada e 
escolher os temas na plataforma digital que melhor se encaixava à proposta de aprendizagem da escola.

\section{d) Comunicação Oral:}

Notou-se que comunicação oral é uma das habilidades menos trabalhadas em sala de aula na disciplina em questão, inclusive na escola pública participante desta pesquisa, por várias razões que vão desde problemas estruturais, como o número elevado de alunos por turmas até o fato de haver pouco tempo de aula para essa disciplina ao longo da Educação Básica, com apenas duas aulas por semana.

Desse fato, repercutem consequências abarcando a formação docente na área, visto que é consenso que muitos professores não dominam a habilidade oral referente à língua ensinada e, como decorrência, a ação de expressar suas ideias oralmente representa um grande desafio nas aulas de Língua Inglesa, tanto para alunos quanto para professores.

\section{e) Gamificação:}

As aulas foram gamificadas tanto no formato analógico quanto digital. Na gamificação analógica foi possível criar atividades utilizando cartas, tabuleiros ou dinâmicas presenciais para obter resultados concretos, assim como está na natureza de toda gamificação. Não foi apenas uma ação de entretenimento, e sim algo que promova impactos positivos no "mundo real do aluno". Já na gamificação digital forma aproveitadas as estruturas tecnológicas para criar uma experiência ainda mais envolvente com o aplicativo Duolingo.

\section{f) Engajamento:}

Os alunos eram enturmados e acostumados com a presença do pesquisador - observador- participante - em sala de aula e agiram normalmente durante as aulas. A Conversation e Feedback Station possibilitaram ao pesquisador uma aproximação entre os alunos, com conversas informais e interativas, o que contribuiu para conhecer melhor cada aluno. 


\section{g) Micro-learning por estações:}

O principal objetivo do microlearning nas estações foi conseguir que os alunos consumissem rapidamente um conteúdo e pudessem aplicar o novo conhecimento ou habilidade na prática para reforçar o que foi aprendido. O grande destaque neste critério foi o desafio para os professores ao realizar a organização no planejamento e na correta escolha do foco que foi dado em cada estação. Para que isto pudesse acontecer, e que pequenas doses de aprendizagem pudessem fazer sentido para os alunos em cada estação e para a aprendizagem global das aulas, cada conteúdo teve um tema central a ser desenvolvido por dia.

\section{h) Organização do espaço físico de sala de aula:}

Este indicador visou organizar previamente a sala de aula para os espaços das estações e transformá-las em locais interativos. Assim, sugere-se ter uma Sala interativa (lousa interativa digital; notebooks; mesas redondas; cadeiras; materiais lúdicos e pedagógicos como: livros didáticos, dicionários; cartolina, papel sulfite para as atividades; materiais concretos sobre o tema do dia, conexão com internet banda larga, celular, Duolingo app).

\section{i) Bring your on device (Traga seu próprio dispositivo):}

Um dos principais motivos por essa escolha dos smartphones foi viabilizar a adoção de tecnologia na rotina de estudos, sem a necessidade de fazer altos investimentos de compra, manutenção e atualização de hardwares. Outro ponto forte foi a oportunidade de engajamento dos estudantes nas atividades propostas, dando um novo destino aos celulares e tablets dos discentes, rompendo com a visão de que utilizar esses equipamentos iria dispersar a aulas e os estudantes iriam acessar conteúdos impróprios, envolvendo também a segurança e o controle. Um ponto positivo nesta abordagem foi criar diferentes espaços dentro e fora da sala de aula, pois o BYOD possibilitou que a aprendizagem ocorresse em outros espaços como biblioteca, jardim, áreas externas, além da sala de aula 
das turmas trazendo mais dinâmica e mobilidade nos espaços já existentes da escola durante a aulas de inglês.

\section{j) Inclusão:}

As práticas foram inclusivas, pois favoreceram a diversidade à medida que considerou que todos os alunos podem ter necessidades especiais em algum momento de sua vida escolar. Há, entretanto, necessidades que interferem de maneira significativa no processo de aprendizagem e que exigem uma atitude educativa específica da escola como, por exemplo, a utilização de recursos e apoio especializados para garantir a aprendizagem de todos os alunos.

É uma variedade de inclusão a partir da realidade social, que pode ampliar a visão de mundo e desenvolver oportunidades de convivência a todas as crianças. As práticas propiciaram um espaço para todos inclusive para três alunos autistas e um de baixa visão que realizou as aulas no desktop com letras de tamanho maior.

\section{Discussão}

$\mathrm{Na}$ discussão desse estudo, em função da atividade aplicada, houve um cuidado ao realizar a curadoria educacional para que fosse possível implantar este projeto. Segundo Saad (2012), o professor em sala de aula pode se apropriar dessa tarefa de curadoria digital para aprimorar sua atividade e ajudar os alunos a buscar conteúdos de forma mais segura e autônoma. Garcia e Czeszak (2019) entendem a curadoria como uma forma de organização de conteúdos com foco em projetos. Assim, o professor-curador tem a possibilidade de levar para a sala de aula, de forma organizada, assuntos transversais às disciplinas, traduzidos para a linguagem daquele grupo em questão, com o objetivo de estimular debates. "O curador é uma pessoa que está conectada e sabe se comunicar com o seu público-alvo. Além disso, ele precisa dominar o assunto", afirma a professora. Da mesma forma, o professor-curador será mais efetivo se for multimídia, aliando informações de livros didáticos digitais e impressos, blogs, vídeos e outros suportes. Nesta perspectiva os principais pontos encontrados 
para implementação e para serem desenvolvidos no processo são seguintes conforme items a e b:

\section{a) Pontos importantes para implementação}

- Introduzir arquivos com conteúdos de apoio que sejam diretos e objetivos como uma receita culinária, uma letra de música.

- Montar aulas curtas para facilitra o download e otimizar o tempo online dos alunos.

- Indicar visualmente a duração de cada conteúdo- vídos, games, etc.

- Garantir que as zonas de interatividades (botões) sejam no tamanho adequado, pois os alunos irão utilizar os dedos (o touch) para clicar e não o mouse.

\section{b) Pontos a serem desenvolvidos no processo}

- Melhorar os recursos para o aprendizado do aluno, que poderá contar com um dispositivo computacional para execução de tarefas, anotação de ideias, consulta de informações via Internet, registro de fatos através de câmera digital, gravação de sons e outras funcionalidades existentes;

- Aumentar as possibilidades de acesso ao conteúdo, incrementando e incentivando a utilização dos serviços providos pela instituição, educacional e o que está disponível da internet e em outros aplicativos.

- Preparar o corpo de professores discutindo ass estratégias de aprendizado disponíveis, através de novas tecnologias que dão suporte à aprendizagem formal como à informal;

- Fornecer meios para o desenvolvimento de métodos inovadores de ensino e de treinamento, utilizando os novos recursos de computação e de mobilidade; 


\section{Considerações finais}

Esse estudo, realizado em uma escola pública, utilizando estratégias de ensino híbrido com uso de celulares, evidenciou a necessidade de o professor se preparar para fazer planejamentos sintonizados com a integração de tecnologias móveis, uso de aplicativos, uso de materiais digitais e analógicos, construindo percursos mais responsivos, expansíveis e criativos.

Constatou-se que grande diversidade de funcionalidades e softwares de aprendizagem presentes nos aparatos moveis fazem com que sua adesão às atividades mediadas por tais tecnologias possam se tornar mais atraentes e aplicáveis ao estudante.

Conseguiu-se estabelecer uma lista de indicativos para que possam direcionar outras aplicações de atividades. Os indicativos foram: a) concentração; b) mobilidade; c) Blended Learning (ensino híbrido); d) comunicação oral; e) gamificação; f) engajamento; g) micro learning por estações; h) organização do espaço físico de sala de aula; i) Bring your on device e j) inclusão

Compreende-se que em condições adequadas o indivíduo pode aprender a partir do seu universo de valores, habilidades e competências já dominadas ou a desenvolver. Por outro lado, entende-se que o papel do professor é mediar a aprendizagem, proporcionando um clima positivo por meio da organização dos componentes materiais, intelectuais e emocionais do aprendizado e da disponibilização dos recursos, além de compartilhar sentimentos e pensamentos com os aprendizes, mas sem dominação.

Observa-se que é preciso considerar a necessidade de criar espaços para a discussão, para as trocas, para a reflexão, enfim, para o desenvolvimento de ambientes de aprendizagem centrados em metodologias ativas e colaborativas valorizando a interação social e a autonomia dos alunos. Verificou-se que, ao proporcionar um ambiente no qual o aluno possa elaborar, expressar e compartilhar suas ideias, por meio de interações espontâneas, entre seus pares, o professor favorece o aprendizado colaborativo. 
A mediação com mobile learning neste estudo possibilitou a co-criatividade no processo de ensino e aprendizagem, na medida em que enfatizam o trabalho em grupos, nos quais alunos traçam e buscam atingir um objetivo e consequentemente adquirem novos conhecimentos linguísticos. Essa interação que ocorre entre os estudantes e professores constitui o alicerce da aprendizagem colaborativa, cuja finalidade é aprimorar as habilidades deles para as atividades cooperativas em equipe, processos interpessoais de comunicação e utilizar a experiência cultural da inteligência coletiva.

\section{Referências}

BACICH, L. MORAN, J. (Orgs.). Metodologias ativas para uma educação inovadora:uma abordagem teórico-prática [recurso eletrônico]. Porto Alegre: Penso, 2018. e-PUB.

BACICH, L.; TANZI NETO, A.; TREVISANI, F. M. Ensino híbrido: personalização e tecnologia na educação. Porto Alegre: Penso, 2015.

CAMPBELL, D.F.J. Mode 3 Knowledge Production in: Quadruple Helix Innovation Systems. Springer Briefs in Business 7, 2012. Disponível em: ttps://www.springer.com/cda/content/document/cda_downloaddocument/ 9781461420613-c1.pdf?SGWID=0-0-45-1263639-p174250662. Acesso em 10 set. 2020 .

CECHINEL, C. Modelos de Curadoria de recursos educacionais digitais. Santa Catarina, 2017. Acesso em: http://www.cieb.net.br/wp-content/uploads/2017/10/CIEB-Estudos-5-Modelos-de-curadoria-de-recursos-educacionais-digitais-31-10-17.pdf

CORRÊA, Elizabeth Saad; BERTOCCHI, Daniela. O Algoritmo Curador: $O$ Papel do Comunicador num cenário de curadoria algorítmica de informação. São Paulo, ECA, USP, 2012.

FREDRICKS, J. A., \& MCCOLSKEY, W. The Measurement of Student Engagement: A Comparative Analysis of Various Methods and Student Self-report Instruments. In Christenson, Sandra L. 2012.

FILATRO, A. Design Instrucional na Prática. Pearson Education do Brasil, 2018. 
GARCIA, M.S.S. CZESZAK, W. Curadoria Educacional: práticas pedagógicas para tratar o excesso de informação e fake news em sala de aula. São Paulo: editora Senac, 2019.

GARCIA, M. S. S. Design de Aplicativos Mobile para a Aprendizagem de Língua. EaD Em FOCO, 6 (1), 2016. doi:http://dx.doi.org/10.18264/eadf.v6i1.371

LADD, G. W.M. (2009). Continuity and change in early school engagement: Predictive of children's achievement trajectories from first to eighth grade? Journal of Educational Psychology, 101 (1), 190-206. 2009.

MARCONI, Marina de Andrade; LAKATOS, Eva Maria. Técnicas de pesquisa. 4. ed. São Paulo: Atlas, 1999.

MATTAR, J. A. Projeto educacional: educação a distância na prática. São Paulo: Artesanato Educacional, 2014.

MORAN, J. Metodologias ativas para uma aprendizagem mais profunda. In: BACICH, Lilian; MORAN, José (Orgs.). Metodologias ativas para uma educação inovadora: uma abordagem teórico-prática [recurso eletrônico]. Porto Alegre: Penso, 2018. e-PUB

SANTAELLA, L. Linguagens líquidas na era da mobilidade. São Paulo: Paulus, 2007.

STARKER, H.; HORN, M. CHRISTENSEN, C. Blended: usando a inovação disruptiva para aprimorar a educação. Porto Alegre: Penso, 2015.

SOUSA, E. F. D.; VASCONCELOS, T. C. O papel do psicopedagogo no contexto atual. Revista Brasileira de Educação e Saúde, 2(1), 53-58. 2013. Disponível em: <http://www.gvaa.com.br/revista/index.php/REBES/article/view/2052>. Acesso em: 26 set. 2020.

WALKER, A., RECKER, M., ROBERTSHAW, M. B., OLSEN, J., SELLERS, L., LEARY, H., KUO, Y.-C., \& YE, L. Designing for problem based learning: A comparative study of technologyprofessional development. Presented at the American Educational Research Association Conference, New Orleans, LA, 2011.

Submetido em: 07-4-2021

Aceito em: 21-7-2021 